\title{
LA ORGANIZACIÓN RED EN EDUCACIÓN A DISTANCIA
}

\author{
(THE NETWORK ORGANIZATIONAL MODEL IN DISTANCE EDUCATION)
}

\author{
Daniel Domínguez Figaredo \\ Universidad Nacional de Educación a Distancia, UNED (España)
}

\section{RESUMEN}

En los últimos años la educación a distancia ha despejado las dudas sobre su viabilidad y consolidado su posición central dentro de la oferta educativa general. Sin embargo, ese proceso de consolidación no se ha dado de una manera clara y abierta para los agentes educativos, sino que ha avanzado en paralelo a la confusión que se deriva del uso de métodos y formas de organización institucional diseñadas para la no presencialidad. Esta situación adquiere una nueva dimensión con el uso masivo de Internet en los procesos didácticos y en la dinámica organizativa. Este artículo analiza el fenómeno de la organización de las instituciones de educación a distancia basada en Internet. A partir de un análisis de las opciones organizativas en los principales modelos institucionales, se profundiza de forma especial en las posibilidades de un tipo de organización reticular aplicado en casos de uso masivo de Internet. El texto compara las diversas formas de organización aplicadas en la educación superior y discute las consecuencias institucionales de apostar por un modelo basado en las propiedades estructurales de las redes.

Palabras clave: educación a distancia, educación superior, gestión organizativa, organización red, Internet, e-learning.

\begin{abstract}
Despite the scepticism that existed in previous years about its viability, distance education now occupies a central position in the education mainstream. Nevertheless, this development has been accompanied by a parallel growth in confusion about the application of methodologies and forms of institutional organization. This article centers on the diverse organizational possibilities for different institutional models of distance education. Our analysis deals with the possibilities of a type of "reticular organization" for institutions based on network communications via the Internet. We examine diverse forms of organization and discuss the implications for institutions that follow a model based on the structural properties of the networks.
\end{abstract}


Keywords: distance education, higher education, management, network organization, internet, e-learning.

\section{LA EDUCACIÓN A DISTANCIA COMO PROCESO SOCIOCULTURAL, DIALÓGICO Y CONSTRUCTIVISTA}

El aprendizaje se sustenta en un proceso de comunicación dialógico que permite estructurar las relaciones entre los agentes implicados -docente y discente-y de éstos con el entorno. La educación es también construcción del conocimiento en contexto. Los agentes integran los conocimientos siguiendo un proceso constructivista y dentro de una lógica que avanza de los elementos sencillos a los complejos (Coll, 1993). De acuerdo con esta visión, una propuesta analítica integradora de los fenómenos educativos debería integrar, al menos, los componentes constructivista -según la cual los objetos con los que interactuar y operar son construcciones de construcciones anteriores (Jones, 2006)-, sociocultural -considerando que la educación es un fenómeno social practicado de acuerdo a códigos culturales-y situado -donde todos los procesos de relación están contextualizados en escenarios determinados, ya sean físicos o virtuales-. Este artículo toma en consideración estas bases analíticas para avanzar en la definición de un marco estructural donde ubicar las diferentes formas de organizar las propuestas educativas en escenarios a distancia.

Como ocurre en las prácticas educativas presenciales, la mejora del proceso comunicativo y la contextualización de las experiencias son elementos clave para mejorar la calidad de la educación a distancia. En este caso, la base para desarrollar aprendizajes de calidad es más amplia cuando la comunicación es de ida y vuelta y alcanza a todos los agentes implicados, teniendo en cuenta su marco de acción. Las tecnologías digitales avanzan en esa dirección y permiten que la educación a distancia enriquezca el diálogo didáctico, proyectando altas dosis de interactividad en las prácticas de aprendizaje. Pero el diseño de procesos educativos altamente dialógicos basados en tecnologías de la información requiere partir de ciertos planteamientos estructurales y organizativos que permitan garantizar la correcta disposición de todos los elementos implicados: docentes, aprendices, administradores, técnicos, etc. En las etapas anteriores a la revolución de las tecnologías de la información los modelos organizativos predominantes en educación a distancia basaban su fortaleza en la jerarquía y la alta estructuración de los procesos. Sin embargo, como consecuencia de su excesiva rigidez y burocratización, los modelos tradicionales presentan numerosas dificultades para adecuarse a los enfoques abiertos y descentralizados de las metodologías online. Los espacios de socialización e interacción basados en tecnologías de la información facilitan el desarrollo institucional y de experiencias 
educativas innovadoras que responden a una visión sociocultural y situada. Pero, para que sea enteramente eficiente, esa disposición demanda un nuevo diseño estructural en las organizaciones de educación a distancia que sea capaz de absorber y canalizar la información que fluye por las redes de comunicación dentro de un marco horizontal y distribuido.

El análisis de las propuestas organizativas en las instituciones con metodologías e-learning o basadas en la telemática (Ortiz, 1998; Zapata, 2001; Wilson, 2002; García, 2004; Arafeh, 2004) -las que desarrollan todas las fases del proceso educativo a distancia, apoyándose en Internet y las tecnologías digitales- evidencia que tales propuestas replican los modelos clásicos de los centros que imparten educación presencial: más jerárquicos si la concepción es cerrada (conductista), y más horizontales si la concepción es abierta (constructivista). Dentro de los primeros, el más habitual es el de tipo burocrático, caracterizado por la rigidez y la fuerte jerarquización. Sin embargo, la adecuación de este modelo a los esquemas de la enseñanza basada en tecnologías digitales y metodologías e-learning no es del todo óptima, sobre todo cuando se pretende sacar provecho del potencial comunicativo de Internet. Entre las diversas alternativas, la que se ha dado en llamar "organización red" se adapta plenamente a las condiciones estructurales del trabajo distribuido apoyado en Internet. Por tanto, puede adecuarse a las necesidades de un tipo de organización descentralizada y que pretende aplicar economías de escala en sus unidades. El modelo de la organización red se caracteriza por delegar las funciones operativas de la organización en células altamente independientes e interconectadas, que garantizan una gran flexibilidad y la adaptación a las necesidades de la institución y los estudiantes.

Para poner en contexto la alternativa de la organización red a los modelos tradicionales se propone comparar los rasgos dominantes en las principales configuraciones organizativas, junto con las implicaciones para las instituciones con métodos de enseñanza basados en sistemas e-learning ${ }^{\mathbf{1}}$. El desarrollo organizativo de la educación a distancia ha discurrido en paralelo a los marcos conceptuales dominantes en la disciplina. Tanto el nivel organizativo como el conceptual han estado íntimamente relacionados con las posiciones (paradigmáticas) sobre la enseñanza y el proceso de mediación tecnológica en educación a distancia. Es por ello que se puede hablar de las diversas formas de organización como algo asociado históricamente a las generaciones de la educación a distancia (Kaufman, 1989; Nipper, 1989). 


\section{INDUSTRIALISMO Y EDUCACIÓN A DISTANCIA}

Las teorías educativas que promueven la autonomía del estudiante fueron las predominantes en la etapa de la institucionalización de la educación a distancia, el momento histórico en el que surgen las grandes megauniversidades (Daniel, 1996). Esa concepción de la enseñanza a distancia hace que aparezcan formas específicas de organización institucional, staff administrativos y disposiciones metodológicas. Las teorías de la autonomía del estudiante asocian la educación y la "enseñanza" desde un planteamiento de "instrucción” y toman como base la especial condición del distanciamiento físico entre profesor y estudiante. El proceso de enseñanza se organiza de una forma individualizadora. El estudiante avanza según su ritmo personal y dentro de unos parámetros básicos establecidos por el docente. El estudio independiente viene a ejemplificar una concepción de la enseñanza que organiza el resto de elementos didácticos en función de la autosuficiencia del discente para adquirir conocimientos.

Para poder regular adecuadamente su aprendizaje, además de los hitos temporales, el estudiante debe disponer de materiales que permitan desarrollar las tareas en condiciones similares a las de la presencialidad, donde el profesor coadyuva para la construcción de conocimientos, y sirve de orientador y facilitador de nuevas situaciones problematizadoras.

Con objeto de dar respuesta a la situación de distanciamiento y promover aprendizajes ricos, las teorías de la autonomía asignan al profesor las tareas principales de elaboración de materiales de aprendizaje y de supervisión del proceso de enseñanza-aprendizaje. Finalmente, este proceso estará dispuesto en torno a tres componentes (Delling, 1966; Moore, 1977): (1) actividades preparatorias, de consideración de necesidades, objetivos y estrategias; (2) actividades ejecutivas, de realización de tareas instructivas, básicamente, supervisión y apoyo al aprendizaje del alumno y; (3) actividades evaluadoras, de valoración, con un evidente carácter cuantitativo, ya sea esta evaluación más o menos procesual y de seguimiento del aprendizaje obtenido. Esas tres tareas conforman en sí la estructura básica de todo modelo pedagógico, pero dentro de un modelo organizativo industrial, definido por hitos y logros formando parte de un proceso cerrado, su funcionalidad está claramente limitada por una acepción marcadamente reduccionista que deja de lado las necesidades comunicativas de todo proceso educativo.

Relacionadas con las teorías del aprendizaje autónomo, en muchos casos las propuestas de industrialización se han convertido en la referencia organizativa 
de las instituciones de educación no presencial, y muy especialmente de las megauniversidades. El modelo de la universidad industrial se fundamenta en las posibilidades de trasvasar los métodos de organización de las empresas a los centros de educación a distancia. En un estudio comparativo realizado por Peters (1973) se comparaban las tipologías organizativas en centros educativos y empresariales, concluyendo con similitudes en algunos conceptos básicos como son, para el caso de la educación a distancia: (1) división del trabajo, realizado por profesores de apoyo; (2) racionalización en la distribución del conocimiento del profesor a los alumnos; mecanización de los procesos; (3) línea de ensamblaje, en la elaboración de materiales didácticos; (4) producción en masa, para una gran cantidad de alumnos; (5) preparación y planificación de los cursos y enseñanzas a impartir; estandarización, con criterios comunes para todo el grupo; (6) cambio funcional, según los roles que adquieren los docentes encargados de los programas formativos; (7) monopolización, con disposiciones internas centralizadas, jerárquicas y autoritarias.

Aparte del carácter eminente reduccionista de estas propuestas, más recientemente han surgido nuevas razones para la crítica de las teorías industriales, basadas en la disparidad organizativa que caracteriza en la actualidad a las empresas y los centros educativos en relación a las propuestas clásicas. A partir de finales de los ochenta del pasado siglo los programas organizativos de las empresas tomaron un carácter más abierto, dinámico y horizontal. Con objeto de mejorar su competitividad, las empresas han tendido a incentivar la creación de conocimiento y la difusión de la información. Para ello han adoptado diversas medidas, como los métodos de trabajo colaborativo aplicados en grupos conformados ad hoc, el desarrollo de estructuras como los "círculos de calidad" en distintos niveles de la organización, o la promoción de formas de liderazgo que permitan a los coordinadores dinamizar con eficiencia los procesos de comunicación. Por ello, puede decirse que los actuales modelos de gestión empresarial difieren considerablemente de las clásicas propuestas fordistas y mecanicistas en que se basaban las teorías de la industrialización pedagógica.

La oposición a las teorías clásicas también se ha fundamentado en las teorías de la comunicación. Desde la consideración de que la mejora de la calidad comunicativa entre el docente y el estudiante redundará en la mejora del proceso didáctico, las teorías de la comunicación plantean el reto organizativo de alcanzar una síntesis provechosa entre la independencia y autonomía del estudiante -que carece de una referencia física donde converger con el resto de agentes educativos- y la necesaria interacción docente-discente. El fin último es el de reducir la "distancia comunicativa" (Bagnall, 1988) entre los agentes educativos, entendiendo que la sola presencia física no es siempre garantía de interactividad significativa y que ésta, 
sin embargo, puede ser conseguida con una adecuada planificación didáctica y a través de la combinación de otros medios tecnológicos distintos a los utilizados en el aula, como son: los materiales de aprendizaje impresos, audiovisuales y digitales, las estrategias de conversación síncrona, los comentarios orales realizados sobre trabajos escritos, las discusiones en grupo, etc. En última instancia, este enfoque persigue que la comunicación mediada en un escenario pedagógico no presencial garantice una alta bidireccionalidad, síncronía e interactividad.

\section{LA BUROCRACIA ORGANIZATIVA EN EDUCACIÓN A DISTANCIA}

El modelo burocrático aplicado a la educación a distancia es la consecuencia de proyectar las teorías de la industrialización al nivel de la estructura organizativa ${ }^{2}$. La organización crea unas estructuras rígidas y fuertemente jerarquizadas, las decisiones se centralizan en el vértice estratégico y los niveles intermedios dela organización, ylos miembros de la organización actúan con escasa autonomía e iniciativa. Por tanto, se produce una gran separación entre los niveles de decisión y los niveles operativos. La estructura organizativa tiende a ser muy vertical, con numerosos niveles jerárquicos intermedios, que muchas veces responden a fines personales antes que organizativos. Los órganos administrativos suelen ser celosos defensores de las competencias que tienen atribuidas y tienden a la autarquía, evitando no cualquier tipo de intromisión sino también la colaboración con otros órganos respecto a lo que, según su forma de ver, sólo a ellos compete. De tal forma, que el nivel operativo está constituido por una pléyade de compartimentos estancos que tienden a un comportamiento autista. En estas organizaciones se mira mucho hacia arriba, raramente hacia los lados y casi nunca hacia abajo (Carmona y Céspedes, 2002).

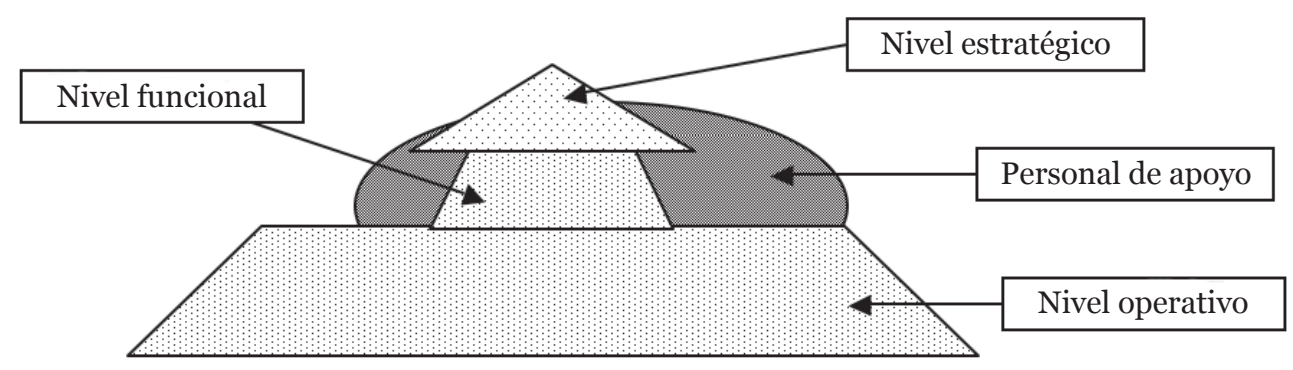

Figura 1. Componentes de la organización profesional o burocracia profesional (Adaptado de García, 2003, p.16o) 


\section{LA ORGANIZACIÓN RED}

Inicialmente, la organización red concibe el ámbito institucional como una metáfora de la sociedad. La "sociedad de la información" (Castells, 2000) se caracteriza por su complejidad, de forma que las jerarquías estatales difícilmente pueden aspirar a resolver, por sus propios medios, todos los problemas que se plantean en ellas, siendo necesario implicar a los diversos agentes funcionales que actúan en las mismas, adoptando una organización interconectada en forma de red. En palabras de Innerarity (2002, p.34), se trata de "sociedades sin vértice ni centro", en las que "sus relaciones ya no son jerárquicas sino heterárquicas, o sea, estructuradas en forma de red", constituyendo esta peculiaridad "su complejidad específica”. Pues bien, lo que Innerarity propugna para la estructura social, es también aplicable para una estructura organizacional.

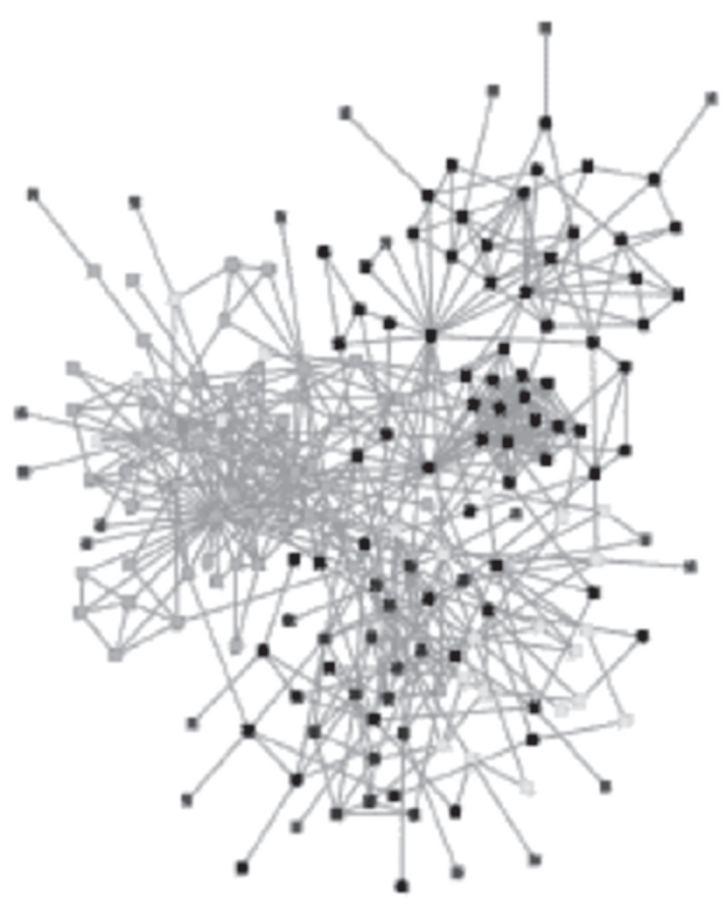

Figura 2. Representación gráfica de una organización red

La configuración del diseño organizativo que corresponde al modelo de organización red se resume muy bien en torno a los siguientes ejes, en los que Internet constituye un elemento indispensable (López y Leal, 2002): (1) Un 
centro relativamente pequeño donde se toman las decisiones estratégicas. En este nivel Internet ayuda a recabar y a gestionar la información para realizar la toma de decisiones. (2) Un conjunto de unidades fuertemente descentralizadas (en el nivel local y en el funcional) encargadas de traducir las estrategias en acciones operativas. En este nivel Internet deberían emplearse para mejorar la actividad interna. (3) Un conjunto de organizaciones paralelas (servicios, bibliotecas, asociaciones de estudiantes, etc.) internas y/o autónomas que se encargan de la gestión e implementación de los programas. En este nivel Internet debería emplearse para mejorar la actividad interna de estas organizaciones. (4) Creación de interfaces de relación institución/usuarios capaces de hacer fáciles y amigables esas relaciones.

La organización red comienza a ser un recurso organizativo dentro de las universidades (Salas, 2002), si bien en la actualidad ninguna aplica plenamente sus principios (Domínguez, 2006 y 2007). En este modelo se constituye un pequeño equipo central -en relación con el tamaño total del sistema- que desarrolla la estrategia y que, para ejecutarla, delega en células o unidades independientes que funcionan coordinadas tanto con la sede central como entre ellas. Las unidades pueden ser subcontratadas (externalización) o internas (células). Entre las características de la organización red destacan su disgregación de funciones y la concentración de esfuerzos, la máxima flexibilidad que permite el sumar o restar partes según sea necesario para satisfacer los objetivos, y la mayor capacidad de innovación en la medida en que forman parte de la organización células que aportan formas de acción dispares.

Una organización que funciona como una red es una organización que colabora, que aborda los problemas de los usuarios con perspectiva integral (porque los problemas no están segmentados), que coordina y es capaz de integrar armónicamente el funcionamiento de sus nodos, que orienta su actuación a conseguir resultados y generar valor interno y para sus usuarios y, por tanto, no teme sino que promueve su participación. 


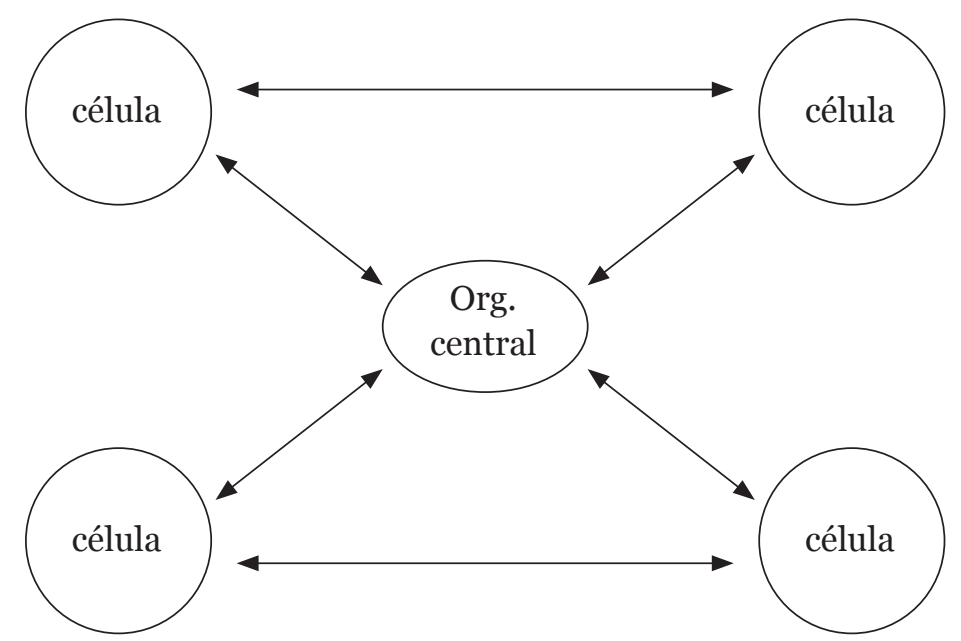

Figura 3. Componentes de la organización red (Adaptado de García, 2003, p.173)

\section{CAMBIO ORGANIZATIVO EN EDUCACIÓN A DISTANCIA: HACIA LA ORGANIZACIÓN RED}

El resultado de implementar diseños organizativos que integran espacios virtuales en el nivel macro -como ocurre en e-learning con los entornos de aprendizaje virtual (EVA) - deriva en una concepción reticular de los vínculos entre las distintas unidades. La organización red -término no análogo al de redes organizacionales- es el paradigma de ese enfoque. Ahora bien, la terminología de redes organizativas e institucionales no es reciente. Si acaso, lo que ha cambiado con las redes informáticas no es tanto el diseño de la organización cuanto la propia tecnología. Hasta ahora el problema era el de coordinar distintos segmentos dentro de una organización, pero con la digitalización es posible tener la flexibilidad de la red y mantener al tiempo la coordinación, unidad de proyecto y toma de decisiones en relación con las tareas que hay que realizar (Castells, 1998). El resultado de esta forma de estructura es la organización red.

\section{Externalización basada en redes}

La organización red funciona mediante unidades o células dispersas interconectadas. La matriz mantiene la unidad estratégica y la unidad jurídica, pero cada sucursal trabaja con gran autonomía. En los casos de externalización, la forma jurídica de este tipo de células puede definirse como fundación, convenio 
de colaboración, contrata u otras formas diversas de acuerdos. Esta constitución jurídica deberá dotar de carta de naturaleza al tipo de sistemas de asociación o consorcio establecido. A modo de ejemplo, Tony Bates (2001) recoge los siguientes tipos de asociación entre instituciones de enseñanza superior y otras corporaciones interesadas: publicidad y marketing conjuntos de cursos en línea; marketing de servicios tecnológicos; transferencia de créditos; valoración de aprendizajes previos; franquicias; planificación conjunta de cursos y programas; desarrollo conjunto de cursos; y acreditación externa. Este punto es realmente importante en el sector de la enseñanza superior. Actualmente, los centros universitarios, las empresas de producción de contenidos, las distribuidoras de materiales educativos y las escuelas de negocios operan parcialmente en red, especialmente para gestionar sus relaciones externas. Mediante vínculos relacionales conforman alianzas estratégicas para lanzar un producto operativo en un mercado concreto. Ese producto puede ser un curso de postgrado, un master o una línea editorial sobre un tema concreto. En los campos ajenos al acuerdo, las instituciones siguen compitiendo por su producto básico y su imagen corporativa. Este fenómeno se denomina unidad de acción en "geometría variable", que no es sino otra red, una red de redes, pues es un producto concreto que se constituye en torno a una red que utiliza las redes que una de las corporaciones tenía (Castells, 1998).

\section{Microgestión institucional basada en redes}

El enfoque de las redes en la organización también revierte en el nivel micro, donde el elemento más característico lo constituye la formación de grupos de trabajo en línea. La creación de grupos de trabajo responde a la tendencia a flexibilizar las organizaciones, así como a la conceptualización de los equipos como procesadores de información (Hinsz, Tindale y Vollrath, 1997). El conjunto de procedimientos telemáticos para el trabajo colaborativo en grupo se denomina groupware y sus posibilidades están en función del tipo de tecnologías empleadas para realizar las tareas ${ }^{3}$ : en el Group Communication Support Systems (GCSS) las tecnologías proporcionan o modifican la comunicación intragrupal a través de Internet; el Group Information Support Systems (GISS) aporta fuentes de información independientes de la comunidad grupal; el Group eXternal Support Systems (GXSS) permite comunicar con personas clave ajenas al grupo; y el Group Performance Support Systems (GPSS), son tecnologías (software) diseñadas para mejorar el rendimiento del grupo de trabajo (Hollingshead, 2001; Rodríguez, 2003). En la práctica, las tecnologías virtuales alteran el funcionamiento de los grupos de trabajo convencionales al menos en cuatro niveles (Peiró y Prieto, 1994): (1) las redes de comunicación permiten interaccionar en el espacio atemporal e incorpóreo del 
ciberespacio; (2) el flujo de la información y la eficacia de la comunicación incorporan las posibilidades de la horizontalidad y transversalidad; (3) la participación de los miembros del grupo se ve alterada por la necesidad de negociar constantemente los significados y, en función del tipo de comunidad virtual -dirigida o no-, el liderazgo se ve sometido a replanteamiento en cada una de las acciones; (4) la toma de decisiones grupal se ve alterada.

Tratando de no caer en un excesivo optimismo, parece que los efectos del uso adecuado de las redes en el entorno organizativo, especialmente de las estrategias de organización mediante groupware, pueden impactar positivamente en dos dimensiones (Dewett y Jones, 2001): (1) eficacia informativa: ahorro de tiempo y costes; y (2) sinergia informativa: mejoras en el rendimiento resultantes de la posibilidad proporcionada por la telemática.

\begin{tabular}{|c|c|c|}
\hline $\begin{array}{l}\text { Características } \\
\text { organizacionales } \\
\text { - } \quad \text { Estructura } \\
\text { - } \text { Tamaño } \\
\text { - } \quad \text { Comunicación } \\
\text { - } \\
\text { - Aprendizaje } \\
\text { - Cultura }\end{array}$ & $\begin{array}{c}\text { TELEMÁTICA } \\
\text { Eficacia informativa } \\
\text { Sinergia informativa }\end{array}$ & \begin{tabular}{ll} 
& \multicolumn{1}{c}{ Resultados } \\
organizacionales \\
- & Eficacia \\
- Innovación \\
- & Conexión entrelas partes \\
& de la organización
\end{tabular} \\
\hline
\end{tabular}

Figura 4. El papel de la telemática en las organizaciones (adaptado de Dewett y Jones, 2001)

Entre las variables apuntadas por Dewett y Jones, destaca una que aporta a Internet un gran valor estratégico y que convierte a esta tecnología en el motor de la organización red: el ahorro de costes cuando se aplica dentro de un proceso de transformación de la comunicación interna ${ }^{4}$. Y considerando la siguiente matización (Fernández, 2004): “no [es] cualquier tipo de comunicación, sino la que permite nuclear equipos de trabajo, que funcionen con propósitos comunes, que aprendan de lo que hacen otros para reinvertirlo en nuevos proyectos". Puesto que los equipos de trabajo operan en un entorno donde sobreabundan los flujos de información, la capacidad de actuar sobre esa información y el conocimiento que se pueda extraer de los datos juegan un papel crucial en la reconstrucción organizativa virtual. En ese sentido, los procesos concretos de trabajo en redes apuntan en la siguiente dirección (Fernández, 2004): 
"Los equipos de trabajo, ya sea que se constituyen como comunidades virtuales o con la denominación que se les quiera dar, requieren manejar, en primer lugar, la información y la experiencia propia en un espacio virtual compartido, transparente, accesible, organizado, donde queden registradas todas las interacciones y donde el conocimiento generado no dependa sólo de sus propias capacidades, sino de sus capacidades para extender la red virtual hasta los confines de sus propios objetivos".

En relación a la posibilidad de implementar estas acciones en el ámbito específicamente universitario, Duart y Sangrá (2000) apuntan una reflexión basada en la experiencia de la Universitat Oberta de Catalunya (UOC). La posibilidad de aportar por modelos integradores insertos en comunidades virtuales de aprendizaje que suponen un espacio de formación compartida, estará en función de los siguientes ejes: (1) accesibilidad, relacionado con las posibilidades de las redes de romper los límites espacio-temporales y de las lenguas; (2) modelo de aprendizaje, hacia disposiciones más creativas en los planteamientos que no reproduzcan miméticamente lo que se ha venido haciendo hasta ahora; (3) modelos de docencia, que afecta al perfil de los profesionales, cada vez más facilitadores que instructores y dentro del entorno de aprendizaje virtual, relacionado con los materiales y los estudiantes; (4) estilo organizativo y cultural, en este caso se trata de conjugar el ideario de la institución, lo que la dota de identidad dentro de la oferta global, de manera que contribuya a enriquecer la diversidad desde el espacio de los flujos; (5) interculturalismo, relacionado con el eje anterior, las instituciones que operan en Internet tienen la posibilidad de relacionar culturas diferentes y fomentar el enriquecimiento entre todas ellas.

Ahora bien, los actuales modelos organizativos de las universidades responden a características propias que hacen difícil implementar en su totalidad estas propuestas integradoras. La bifurcación "docencia" versus "organización" dota a las universidades de un sesgo peculiar. Esa especificidad se plasma en el estudio "Modelos Virtuales de las Universidades Europeas" (Virtual Models of European Universities, 2004, que analiza el uso y potencial de las tecnologías de la información en las universidades europeas. Sus análisis presentan una panorámica muy completa de los logros y limitaciones de las instituciones de enseñanza superior europeas. Las respuestas dadas a la encuesta - con una muestra de más de 200 universidades- dieron lugar a la división en cuatro grupos de universidades en lo que se refiere al uso actual de las nuevas tecnologías en el marco organizativo y docente: (1) universidades punteras (18\%), que se distinguen por su superioridad en todos los sentidos, incluido su nivel de cooperación con otras universidades 
e instituciones educativas; (2) universidades centradas en la cooperación (33\%), que se caracterizan por su gran implicación en la cooperación estratégica con universidades locales y extranjeras, así como con otras instituciones educativas; al igual que las punteras, han avanzado mucho en cuanto a la integración de las nuevas tecnologías en la docencia desarrollado en el campus, pero presentan un uso mucho más limitado de cursos de e-learning y de servicios digitales; (3) las universidades autosuficientes (36\%) son el grupo más numeroso, y presentan un nivel de integración de las tecnologías en el marco organizativo y educativo parecido al de las universidades del grupo anterior, pero su grado de implicación en la cooperación estratégica con otras universidades o instituciones educativas es mínimo; y (4) universidades escépticas (15\%), que van a la zaga de las demás en casi todas las dimensiones, caracterizándose por un uso limitado de servicios digitales, una escasa integración de las nuevas tecnologías en la docencia en el campus y una proporción muy baja de cursos de e-learning ${ }^{5}$. Los datos de este estudio poseen la virtualidad de tratar conjuntamente los dos componentes que dificultan la integración organizativa en redes, el organizativo y el docente. La mejora en ambos componentes no depende de los usos tecnológicos. Más bien guarda relación con las competencias y las tareas funcionales asignadas a los equipos directivos en la universidad. Es decir, antes que como consecuencia directa de las aplicaciones tecnológicas, las mejoras se producirán con mayores garantías cuando las tareas se integran en un modelo de trabajo en red adecuadamente diseñado y que responda a las finalidades de la organización. La tecnología puede apoyar el cambio en las prácticas, pero por si misma no es determinante para que se produzcan cambios efectivos en el comportamiento de la organización.

\section{¿HACIA LA UNIVERSIDAD RED?}

La tendencia favorable al uso de estrategias de e-gestión en la universidad es alcista, y también es creciente la orientación hacia los modelos de organización virtuales. Tomando como base la evolución en el ámbito empresarial y de las organizaciones online que operan en Internet, es posible avanzar las líneas básicas de la estrategia institucional para la implantación de un modelo organizativo reticular. Las organizaciones en red y los procesos de relación con los usuarios (comunicación externa) y los miembros del staff (comunicación interna) serían los dos vectores esenciales en dicho modelo estructural. 
D. Domínguez

La ORganización RED EN EduCACión a DisTancia

\section{Imitando modelos reticulares: benchmarking empresarial aplicado a la educación a distancia}

Las organizaciones que mejor se adaptan a un escenario operado tecnológicamente son aquellas que introducen altas cotas de flexibilidad y disponen de una estructura reticular, en la que sus células actúan como nodos de una red con unidad de acción común en el centro, donde tiene lugar la toma de decisiones estratégicas, y un alto grado de independencia en las zonas distales. Si el nuevo modelo económico se define como el de las organizaciones que funcionan con y a través de Internet, la universidad, considerada una institución central en las sociedades avanzadas, debería tener acomodo en esa definición.

Castells (2001) emplea dos ejemplos de organizaciones adaptadas a las formas de producción y trabajo en el nuevo sistema tecnológico y en el contexto de la nueva economía: el modelo Modelo Cisco Systems y el Modelo Zara. En el primer caso, el 90\% de las ventas de la empresa y de sus transacciones se hacen mediante la Web de los proveedores de la empresa y los clientes, sin que la empresa haga nada más que poner la ingeniería, poner la Web, actualizarla cada hora, garantizar calidad y organizar la red de proveedores. Es decir, la empresa número uno del mundo en venta de equipamientos de telecomunicaciones del backbone de Internet en el mundo, de routers y switches (enrutadores y conmutadores), apenas posee oficinas físicas y sólo produce sus componente físicos con un par de fábricas en el mundo. El segundo ejemplo es el de la empresa Zara. Esta empresa textil posee cientos de tiendas dispersas en sesenta países, todos ellas conectados en red con la sede central en La Coruña (España). Los gestores de las tiendas envían periódicamente, en intervalos de unos pocos días, informes de evolución de ventas a la sede central donde los diseñadores procesan los datos y determinan las tendencias de mercado, y las comunican directamente a las fábricas que cortan los patrones y producen la ropa. Este sistema de comunicación red permite disponer en las tiendas de cualquier parte del mundo de ropa "actualizada" y "rediseñada" cada dos semanas. La conclusión de Castells es que Internet está cambiando el modelo de empresa, de un modelo fordista basado en la producción estándar, las cadenas mecánicas de montaje y el control de parámetros asociados a la producción, a otro en red, "capaz de articular directamente el mercado, insumos y proveedores y organización interna de la empresa online en todas las tareas" (Castells, 2001). La universidad contemporánea es una institución que realiza la mayor parte de sus tareas relacionadas con la información, por lo que sus procesos de trabajo tienden a relacionarse con los de otros operadores directamente vinculados al procesamiento de informaciones. Esto no quiere decir que los modelos sean equiparables, pero 
sí que organizaciones como la universidad o los servicios integrados en su seno pueden adecuar los macromodelos organizativos de las empresas en red y trasladar sus rasgos principales a la idiosincrasia de un centro formativo de nivel superior. Este nuevo modelo puede traducirse en procesos y estrategias innovadoras, como la flexibilización de las tareas, el desarrollo de acciones con los usuarios adaptándose a sus demandas o, principalmente, la delegación de procesos en las herramientas virtuales, entendiendo que éstas intersectarían las diferentes áreas de la estructura organizativa.

Una disposición de ese tipo responde al modelo propuesto por Bates para introducir a las universidades en las nuevas tecnologías. Según Bates (2001, p.23):

"se recomienda una estructura organizativa que conjugue una mezcla de estrategias centralizadas y descentralizadas. La mezcla dependerá en cierta medida del tamaño de la organización (...) la mayoría de las instituciones necesitarán también de servicios descentralizados con equipamiento técnico especializado y personas especialistas que trabajen con las diversas facultades y departamentos".

En lo que constituye un claro ejemplo de encuadre de los servicios académicos y administrativos en el seno de universidades que operan significativamente con nuevas tecnologías. Y perfila una estructura óptima para un tipo de organización basado en redes de comunicación y trabajo.

\section{Comunicar... en red}

"La historia muestra que la introducción de nuevas tecnologías generalmente va acompañada de unos cambios importantes en la organización del trabajo" (Bates, 2001, p.19). Algunos de esos cambios necesarios ya han sido planteados en este trabajo, pero hay otros igual de importantes relacionados con los procesos de comunicación dentro de la organización y con los agentes externos en los que conviene profundizar para tener una visión global de la lógica de la red en las organizaciones ${ }^{6}$.

Por un lado, las universidades y centros de educación a distancia se han caracterizado hasta fechas recientes por una mezcla de formas de organización agrarias e industriales, con unas estructuras y unos procedimientos jerárquicos, burocráticos y relativamente inflexibles. En cambio, las nuevas tecnologías están asociadas con las formas de organización postindustriales -como la organización 
en red-, que se sustentan en trabajadores muy cualificados y flexibles con un alto grado de autonomía y organizados en unidades operativas relativamente pequeñas y ágiles (Gil y Barrasa, 2003). Estas formas organizativas introducen el discurso de un modelo de trabajo en grupos pequeños que se organizan en función de tareas y áreas de acción y están soportados en redes telemáticas, como el groupware. El trabajo en grupo funciona cuando existe una relación fluida entre los miembros y de el grupo en su conjunto con la estructura orgánica (Rice y Gattiker, 2001), aspecto éste que se ve favorecido en buena medida por las tecnologías digitales.

Por otro lado, entre las características que definen una universidad postfordista, Bates (2001) incluye dos que son capitales para la misión institucional y que guardan relación con el tipo de relación establecida con los agentes externos. Las organizaciones posfordistas presentan: (1) unos productos a medida del cliente y unos servicios diseñados y adaptados a sus necesidades individuales; y (2) unos trabajadores relacionados directamente con los clientes, con una retroalimentación rápida e inmediata para modificar los productos y los servicios ${ }^{7}$.

Las universidades poseen dos tipos de usuarios externos, los estudiantes y las empresas, con los que habrán de establecer lazos relacionales de confianza mutua. Tanto la adecuación del servicio al usuario como la retroalimentación con inputs procedentes de éste, pueden llevarse a cabo mediante sistemas telemáticos, lo que evita disponer de trabajadores "liberados" para la atención al público, y permite que todos se integran en un escenario de relación interna-externa directa mediado telemáticamente.

La implantación en la Universidad de Internet y otras innovaciones tecnológicas que tienen influencia en el modelo de comunicación, determina de forma importante la configuración de la estructura organizacional, haciendo que ésta derive hacia modelos más próximos a una configuración en red que a aquellos caracterizados por la rigidez y verticalidad.

\section{CONCLUSIONES: HACIA UNA ORGANIZACIÓN RED EN EDUCACIÓN A DISTANCIA}

El uso extenso de Internet y la necesidad de adaptarse al cambio, está dando lugar a una serie de ajustes en los procesos organizativos y los modelos pedagógicos que sustentan las instituciones de educación a distancia. Los cambios en los procesos que son fruto de la mediación tecnológica afectan a la estructura de la organización 
y hacen que ésta deba reconfigurarse con el objetivo de mantener su eficiencia. Las necesidades de adaptación afectan a la gestión de los procesos tanto en los ámbitos administrativos como docentes. En última instancia, el cambio estructural puede conllevar la necesidad de redefinir la visión y la estrategia de la institución, así como el modelo de relación de la organización con sus públicos potenciales.

En este artículo se han analizado los principales componentes del modelo de organización red, que se considera la alternativa más adecuada a los formatos tradicionales. Su funcionalidad procede de la similitud entre los principios que lo sustentan y el tipo de trabajo asociado al uso de tecnologías digitales. La descentralización de procesos, la movilidad, la base comunicativa bidireccional o la prevalencia de los flujos y los procesos frente a los productos, y los resultados tangibles, son algunos de los factores que hacen de la organización red una opción adecuada ante las necesidades estructurales de la educación a distancia.

A partir de una concepción de la educación a distancia como proceso sociocultural, dialógicoy constructivista, se han analizadolos aportes delas teorías del industrialismo y de la burocracia organizativa, los referentes más inmediatos en la organización institucional de la educación a distancia. A continuación se han analizado los componentes de la organización red, junto con los cambios que se están produciendo en las universidades a distancia que justifican su introducción en ese ámbito. En el terreno aplicado, la universidad red se caracteriza por ser un ecosistema abierto que actúa de manera descentralizada, con una organización basada en células que colaboran entre sí y que basan su potencial en el diálogo permanente que mantienen con el interior y el exterior. El fuerte liderazgo institucional, la microgestión interna basada en redes y la identificación de los flujos de comunicación son básicos para el funcionamiento de este modelo, que puede ayudar a las instituciones de educación a distancia a adaptarse los cambios que se producen como consecuencia de la digitalización de los procesos en todos los niveles de la organización.

\section{NOTAS}

1 Dado que el ámbito de desarrollo natural de esta forma de organización ha sido el de las instituciones de educación superior, las reflexiones en el conjunto del artículo serán referidas a ese nivel educativo, en el bien entendido que tanto la organización red como el resto de modelos presentados pueden ser implementados con las adaptaciones necesarias en otros tantos contextos educativos (o empresariales).

2 "La estructura organizacional se refiere a todas aquellas formas en las que las organizaciones fragmentan el trabajo en diferentes tareas (diferenciación) para luego integrarlas entre sí (integración)” (García, 2003, p.137). La estructura define 
cómo se dividen, agrupan y coordinan formalmente las áreas de trabajo para que la organización logre sus objetivos (Robbins, 1998). De otrolado, en estetexto se considera el significado del "diseño organizacional" como la descripción de la apariencia de la organización -el otro significado posible se refiere al proceso de establecimiento o cambio de la organización-, que tiene entre sus variables la estructura organizacional (Hodge, Anthony y Gales, 1998).

3 Existen algunas distinciones significativas entre el "trabajo colaborativo" mediante redesinformáticas (ComputerSupported Collaborative Work, CSCW)yel "aprendizaje colaborativo" en EVAs (Computer Supported Collaborative Learning, CSCL). La metodología que soporta el trabajo colaborativo se conoce como groupware y consiste en una estrategia para coordinar actividades de comunicación, colaboración, coordinación y negociación a fin de aumentar la productividad en una organización. Groupware es una metodología colaborativa, pero vinculada a la consecución de objetivos específicos en la organización. Por su parte, el aprendizaje colaborativo es el proceso que ponen en práctica las comunidades virtuales que persiguen algún tipo de conocimiento; parte de los enfoques piagetianos y vygotskyanos basados en la interacción social y se define como el conjunto de métodos y estrategias apoyados en tecnologías que persiguen que cada miembro del grupo sea responsable de su aprendizaje y del de los restantes miembros del grupo. La base de este tipo de aprendizaje está asociada a los términos: interdependencia positiva, interacción, contribución individual y habilidades personales y de grupo (Coleman, 1997; Lucero, 2003).

4 Si bien en las organizaciones de carácter productivo pueden obtenerse rebajas en los costes globales, en las instituciones educativas, la posibilidad de ahorre de costes utilizando nuevas tecnologías parece ser un mito. Para Bates (2001), la tecnología puede mejorar la eficacia de los costes del funcionamiento de la enseñanza superior de diversas formas, pero no es previsible que reduzca costes absolutos.

5 El estudio incluye otros datos interesantes: nueve de cada diez universidades europeas cuentan con Intranets que ofrecen información, aunque sólo cinco de cada diez ofrecen servicios digitales interactivos; casi todas las universidades prevén disponer de una Intranet próximamente y la gran mayoría han elaborado planes para ofrecer servicios digitales; la escasez de recursos financieros es una barrera para progresar hacia el uso generalizado de las nuevas tecnologías y el e-learning; falta una estructura global de apoyo para el e-learning; las actuales estructuras organizativas universitarias parecen impedir la integración del las tecnologías y el e-learning; faltan sistemas para asegurar la calidad de las nuevas experiencias con tecnologías digitales. El estudio data de 2004, pero la situación es muy similar en la actualidad: en un momento de incremento cuantitativo en la dotación tecnológica, los problemas se han trasladado a la definición de líneas estratégicas (en los niveles institucional y metodológico) para definir cualitativamente el uso de esas tecnologías.

6 En este trabajo comunicación interna se considera como "el patrón de mensajes compartidos por los miembros de una organización” (Gil y Barrasa, 2003, p.341). Por su parte la comunicación externa se dirige a establecer relaciones relevantes con el ambiente, del que forman parte otras organizaciones. Ambas están relacionadas: los procesos de comunicación interna se dirigen al establecimiento de una estructura organizacional y a la estabilidad, mientras que los canales de la comunicación externa 
se dirigen a la innovación y la identificación de líneas útiles para el desarrollo actual y futuro de la organización.

7 El resto de características que define una organización postfordista son (Bates, 2001): una gran dependencia de las tecnologías de la información; unos trabajadores a los que se estimula para que creen y desarrollen nuevos conocimientos y formas nuevas de hacer las cosas, o que transformen y modifiquen la información preexistente; unos trabajadores descentralizados, con poder y creativos, que suelen trabajar en grupo; unos trabajadores "nucleares", bien pagados, bien formados y con estudios, con contrato, muchas veces con participación en la empresa mediante opciones de acciones, y muy móviles, además de trabajadores y funciones "no nucleares", que suelen ser subcontratados y carecer de un empleo estable; un fuerte liderazgo, caracterizado por una visión y unos objetivos claros y amplios, y una gestión por parte de expertos, que desempeñen un papel integrador, coordinador y facilitador; muchas veces, una organización especializada y a pequeña escala dependiente de asociaciones y alianzas con otras organizaciones con competencias afines y complementarias; un desarrollo y un cambio rápidos; y operaciones globales. Evidentemente, Bates introduce un factor corrector para el caso de las universidades, en tanto que instituciones específicamente formativas, y concluye que la tendencia es hacia la compatibilidad de determinadas características de una universidad tradicional con el nuevo entorno postfordista.

\section{REFERENCIAS BIBLIOGRÁFICAS}

Arafeh, S. (2004). The Implications of Information and Communications Technologies for Distance Education: Looking Toward the Future. Final Report, SRI International \& American Institutes for Research. SRI Project Number P11913. [en línea] Disponible en: http://www.sri.com/policy/csted/ reports/sandt/it/Distance_Ed_Lit_ Review_FINAL_6-9-04.pdf [consulta 2008, 12 de julio]

Bagnall, R. G. (1988). Reconceptualising and revaluing distance education through the perspective of self-direction, en: Sewart, D.; Daniel, J., Developing Distance Education. Oslo: International Council for Distance Education, 93-95.

Bates, T. (2001). Cómo gestionar el cambio tecnológico. Estrategias para los responsables de centros universitarios. Barcelona: Gedisa.

Carmona, E.; Céspedes, J. J. (2002). Estructuras Organizativas para la innovación. madrid+d revista, 11. [en línea] Disponible en: http://www. madrimasd.org/revista/revista11/ tribuna/tribunas1.asp [consulta 2007, 2 de enero]

Castells, M. (1998). Globalización, tecnología, trabajo, empleo y empresa. La factoría, 7. [en línea] Disponible en: http://www. lafactoriaweb.com/articulos/castells7. htm [consulta 2003, 3 de octubre]

Castells, M. (2000). La era de la información. Vol. I La sociedad red. Madrid: Alianza Editorial.

Castells, M. (2001). Internetyla Sociedad Red. La Factoría, 14-15. [en línea] Disponible en: http://www.lafactoriaweb.com/ default-2.htm [consulta 2002, 25 de abril]

Coleman, D. (1997). Groupware: Collaborative Strategies for Corporate LANs and Intranets. New Jersey: Prentince Hall. 
Coll, C. (1993). Aprendizaje escolar y construcción del conocimiento. Buenos Aires: Paidós.

Daniel, J. (1996). Megauniversities and knowledgemedia:Technologystrategies for higher education. Londres: Kogan Page.

Delling, R. M. (1966). Versuch der Grundlegung $\mathrm{zu}$ einer systematischen Theorie des Fernunterrichts, en: Sroka, L. (Ed.), Fernunterricht 1966, Ferstchrift zum 50 Geburtstag von Walter Schultz-Rahe. Hamburgo: Hamburger Fernlehrinstitut, 23-44.

Dewett, T.; Jones, G. R. (2001). The role of information technology in the organization: a review, model, and assessment. Journal of Management, 27, 313-346.

Domínguez, D. (2006). E-alfabetización y acceso a la formación virtual en la universidad, en: Casado, R. (Coord), Claves de la alfabetización digital. Barcelona: Ariel, 315-334. [en línea] Disponible en: http://www.fundacion. telefonica.com/forum/Alfabetizacion/ pdf/BVIII_3.pdf [consulta 2008, 13 de julio]

Domínguez, D. (2007). Estructuras reticulares, fractales y organización de instituciones de educación a distancia. Ciberalia, 15 de abril. [en línea] Disponible en: http://ciberalia.blogspot. com/2007/04/estructuras-reticularesfractales-y.html [consulta 2008, 13 de julio]

Duart, J. M.; Sangrá, A. (2000). Formación universitaria por medio de la Web: un modelo integrador para el aprendizaje superior. En: Duart, J. M.; Sangrá, A. (Comp.). Aprender en la virtualidad. Barcelona: Gedisa y UOC, 13-49.

Fernández, L. A. (2004). Malo va el que arrastran, aunque vaya en serón nuevo. Enredando, Editorial 431. [en línea] Disponible en: http://enredando. com/cas/editorial/enredando431.html [consulta 2004, 10 de julio]

García Aretio, L. (2004). Blended Learning, ¿es tan innovador?. BENED, Editorial, septiembre. [en línea] Disponible en: http://www.uned.es/catedraunescoead/editorial/p7-9-2004.pdf [consulta 2008, 10 de julio]

García, M. (2003). Estructuras organizacionales. En: Gil, F.; Alcover, C. M.(Coords.),Introduccióna la psicología de las organizaciones. Madrid: Alianza, 137-178.

Gil, F.; Barrasa, A. (2003). Comunicación en las organizaciones. En: Gil, F. y Alcover, C. M. (Coords.), Introducción a la psicología de las organizaciones. Madrid: Alianza, 321-354.

Hinsz, V. B.; Tindale, R. S.; Vollrath, D. A. (1997). The emerging conceptualization of groups as information processors. Psychological Bulletin, 121 (1), 43-64.

Hodge, B.; Anthony, W.; Gales, L. (1998). Teoría de la organización. Madrid: Prentice-Hall.

Hollingshead, A. B. (2001). Communication technologies, the Internet and group research. En: Hogg, M. A.; Tindale, R. S. (Eds.), Blackwell handbook of social psychology: group processes. Malden: Blackwell Publishers, 557-573.

Innerarity, D. (2002). La transformación de la política. Madrid: Ediciones Península.

Jones, K. (2006). A Biographic Researcher in Pursuit of an Aesthetic: The use of artsbased(re)presentationsin "performative" dissemination of life stories. Qualitative Sociology Review, II (1), 66-85.

Kaufman, D. (1989). Third generation course design in distance education, en: Sweet, R. (Ed.), Postsecondary distance education in Canada. Policies, practices and priorities. Athabasca: Athabasca University Press/Canadian Society for Studies in Education, 51-73.

López, J.; Leal, I. (2002). e-Gobierno. Gobernar en la Sociedad del 
Conocimiento. Oñate: Instituto Vasco de Administración Pública.

Lucero, M. M. (2003). Entre el trabajo colaborativoyelaprendizajecolaborativo. Revista Iberoamericana de Educación, sección lectores. [en línea] Disponible en: http://www.campus-oei.org/revista/ deloslectores/528Lucero.PDF [consulta 2003, 8 de noviembre]

Moore, M. (1977). On a theory of independent study. Hagen: Fern Universität.

Nipper, S. (1989). Third generation distance learning and computer conferencing. En: Mason, R.; Kaye, A., Communication, computers and distance education. Oxford: Pergamon, 51-72.

Ortiz, J. R. (1998). La educación a distancia en el umbral del nuevo paradigma telemático. Comunicación en el $I V$ Congresso RIBIE, Brasilia. [en línea] Disponible en: http://lsm.dei.uc.pt/ ribie/docfiles/txt200342415634191.PDF [consulta 2008, 12 de julio]

Peiró, J. M.; Prieto, F. (1994). Telematics and organizational structure and processes: an overview. En: Andriessen, J. E.; Roe, R. A. (Eds.). Telematics and work. Hove: Lawrence Erlbaum Associates, 175-203.

Peters, O. (1973). Die didaktische Struktur des Fernunterrichts. Weinheim: Beltz.

Rice, R. E.; Gattiker, U. E. (2001). New media and organizational structuring. En: Jablin, F. M. y Putnam, L. L. (Eds). The new handbook of organizational communication. Thousand Oaks, CA: Sage, 544-581.

Robbins, S. P. (1998). Comportamiento organizacional. Conceptos, controversias, aplicaciones. México: Prentice-Hall.

Rodríguez, F. (2003). Influencia de las nuevas tecnologías sobre el comportamiento organizacional. En: Gil, F.; Alcover, C. M. (Coords.). Introducción a la psicología de las organizaciones. Madrid: Alianza Editorial, 179-200.

Salas, I. (2002). ¿Por qué la UOC puede concebirse como una organización fractal? Capital Humano, 158. [en línea] Disponible en: http://www.uoc. edu/web/esp/art/uoc/isalaso902/ isalaso902.html [consulta 2008, $13 \mathrm{de}$ julio]

Wilson, B. G. (2002). Trends and Futures of Education: Implications for Distance Education. Quarterly Review of Distance Education [en línea] Disponible en: http://carbon.cudenver.edu/ bwilson/ TrendsAndFutures [consulta 2008, 12 de julio]

Zapata, M. (2001). Formación abierta y a distancia a través de redes digitales: Modelos de redes de aprendizaje. RED: Revista de Educación a Distancia, 1. [en línea] Disponible en: http://www. um.es/ead/red/1/modelos.pdf [consulta 2008, 10 de julio] 


\section{PERFIL ACADÉMICO Y PROFESIONAL DEL AUTOR}

Daniel Domínguez Figaredo. Doctor en Filosofía y Ciencias de la Educación por la UNED (España). Actualmente, es docente e investigador en el Departamento de Teoría de la Educación y Pedagogía Social de la citada universidad. Sus líneas de investigación se centran en analizar las implicaciones educativas de las tecnologías digitales y las dinámicas socioeducativas en el ciberespacio. Más información en http://ddominguez1.googlepages.com/

E-mail: ddominguez@edu.uned.es

DIRECCIÓN DEL AUTOR:

Daniel Domínguez Figaredo

UNED - Facultad de Educación

Senda del Rey, 7, desp. 225

28040 Madrid (Spain)

T: $(+34) 913986356$

Fecha de recepción del artículo: 03/03/08

Fecha de aceptación del artículo: 07/07/08 